\title{
Simultaneous occurrance of papulonecrotic tuberculid and extra pulmonary tuberculosis (cervical lymphadenopathy) in an adult man. A Case report
}

\section{Th Bijayanti Devi, Angila Dorjee}

Department of Dermatology, Venereology and Leprology, Regional Institute of Medical Sciences, Imphal, Manipur, India

Corresponding author: Dr. Angila Dorjee, E-mail: ngla@rediffmail.com

\begin{abstract}
A 35-year-old male presented with recurrent crops of papules and plaques over extensor surface of forearm, elbow, back, face, neck and lower back side of trunk since two months, along with multiple cervical lymphadenopathy over the right side of neck. On the basis of clinic pathological findings and its response to the treatment the diagnosis of papulonecrotic tuberculid (PNT) was made.
\end{abstract}

Key words: Papulonecrotic tuberculid, Extrapulmonary tuberculosis, Erythrocyte Sedimentation rate, Ziehl -Neelsen stain

\section{INTRODUCTION}

Tuberculids was first described by Darrier in 1886, it were originally felt to be related to an allergic response to tubercle bacilli in a patient with tuberculosis at a remote site. It represents an Arthus reaction (type III hypersensitivity reaction) accompanied by delayed-type hypersensitivity reaction (type IV). It is an asymptomatic, chronic disorder, occurring in crops associated with an underlying or silent focus of tuberculosis. The lesions are symmetrically distributed over the extensors of extremities, dorsum of hands and feet, face, and ear [1]. Our patient presented with crops of crusted papulo necrotic lesions on extensor surface of upper limbs, neck, $V$ area of anterior chest and lower lateral side of back. Along with which he had right sided cervical lymphadenopathy for same duration of time. Based on the clinopathological findings, and its response to antitubercular therapy a diagnosis of PNT with was made. We are reporting a case of PNT because it is a rare manifestation even in areas endemic for tuberculosis.

\section{CASE REPORT}

35 years old male shepard by occupation presented to us with crops of mildly itchy papular skin lesion over the extensor surface of forearm and arm, neck, face and lower back since last two months. He gave history of multiple swelling over the right side of neck since last two months. History of fever and generalised weakness was present but no history of cough, weight loss, loose motions, joint pain. No significant past history of any chronic diseases. On cutaneous examination there were multiple excoriated erythematous papules few covered with crust and central necrosis and few skin coloured papules involving the extensor surface of arm, forearm, elbows, face, left lateral side of neck, $\mathrm{V}$ area of neck (Fig. 1). On the lateral side of left lower back the papular lesions were coalesced to form plaque with central necrosis (Fig. 2). Multiple matted firm to hard lymph nodes over the right cervical area (Fig. 1). Oral mucosal was normal, scalp normal, nails were normal.

Investigations revealed raised ESR $75 \mathrm{~mm} / \mathrm{h}$ (Westergren), Mantoux test was strongly positive with induration of $30 \mathrm{~mm}$ X $30 \mathrm{~mm}$. Biopsy from the lesion on the face

\footnotetext{
How to cite this article: Devi TB, Dorjee A. Simultaneous occurrance of papulonecrotic tuberculid and extra pulmonary tuberculosis (cervical lymphadenopathy) in an adult man. A Case report. Our Dermatol Online. 2019;10(2):184-186

Submission: 03.03.2018; Acceptance: 04.12.2018

DOI:10.7241/ourd.20192.20
} 
revealed irregular acanthosis and moderate hyperkeratosis with follicular plugging, the upper dermis shows perifollicular areas displaying geographic necrosis surrounded by palisading histocytes, numerous neutrophils with occasional gaint cells. ZN stain shows no acid fast bacilli (Fig. 3) features were consistent with PNT. Chest X-ray was normal, sputum for AFB was negative.

The FNAC of the cervical lymph node was suggestive of granulomatous lymphadenitis with positive AFB. Rest of the investigations were normal.

Patient was started on Anti tubercular therapy consisting of rifampicin $600 \mathrm{mg}$, isoniazid $300 \mathrm{mg}$, pyrazinamide $1500 \mathrm{mg}$, and ethambutol $800 \mathrm{mg}$ and sunscreens for face and sunexposed area and to follow strict photoprotection. On starting therapy, many

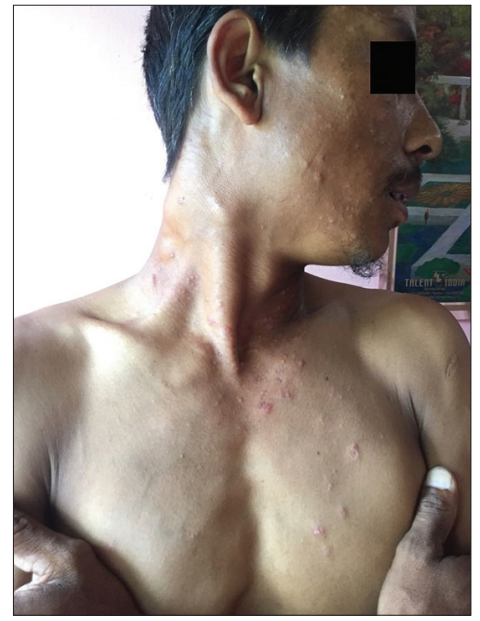

Figure 1: Clinical photograph showing papules, papulopustules lesions with necrotic centre over the face and neck with right sided matted cervical lymphadenopathy

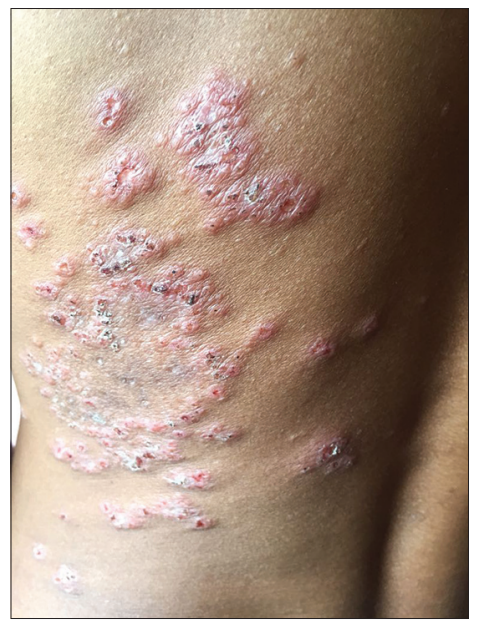

Figure 2: Clinical photograph papular lesions coalescing to form plaque with scales and with few necrotic centre at the back side of lower trunk. lesions on back disappeared within 2 weeks. His general condition has improved and at 4weeks of therapy. He is still on ATT and his skin lesions are healing with scarring at back and trunk.

Prior to the study, patient gave written consent to the examination and biopsy after having been informed about the procedure.

\section{DISCUSSION}

Cutaneous tuberculosis can be a "true" cutaneous tuberculosis like lupus vulgaris, TB verrucosa cutis, scrofuloderma, orifacial TB, miliary TB or tuberculids like papulonecrotic tuberculid, nodular vasculitis, lichen scrofulosorum, erythema nodusum [1-6].

PNT is considered a sign of a good immunological status, because it usually appears in patients with moderate or high degree of immunity [3]. Extracutaneous focus is found in only $30-40 \%$ of cases of cutaneous TB, with cervical lymph nodes being the most common site, as in our patient. Apart from its typical locations on the extensor aspect of the extremities papulo necrotic tuberculid can involve buttocks, face, eyelids and even glans penis [2].

However, strongly positive tuberculin test, suggestive histopathological findings with endarteritis and thrombosis of the dermal vessels; and above all complete remission of the disease after the institution of antitubercular therapy confirmed the condition to be a case of papulo necrotic tuberculid [7]. PNT is a rare manifestation even in areas endemic for tuberculosis [8].

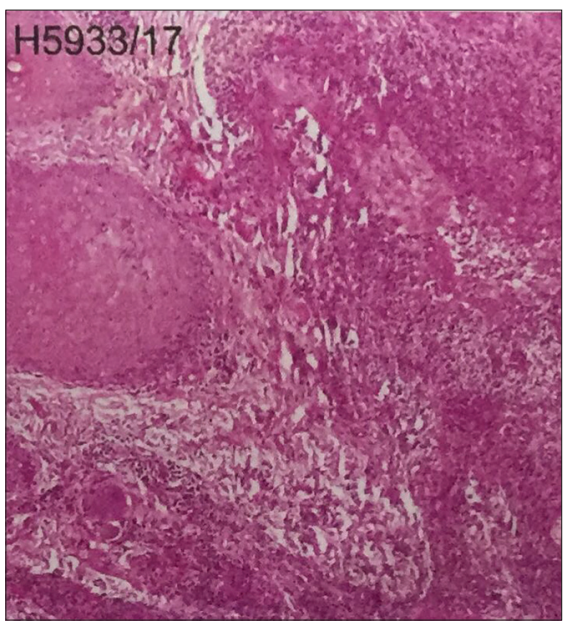

Figure 3: Irregular acanthosis, moderate hyperkeratosis with follicular plugging, dermis shows geographic necrosis in per-follicular areas surrounded by palisading histocytes, numerous neutrophils occasional giant cells. ZN stain no AFB.(Face). (H\&E X 100). 


\section{CONSENT}

The examination of the patient was conducted according to the Declaration of Helsinki principles.

\section{REFERENCES}

1. Bae SH, Yun SJ, Lee JB, Kim SJ, Lee SC, Won YH. Papulonecrotic tuberculid: A rare skin manifestation in a child with mesenteric tuberculous lymphadenopathy. Acta Derm Venereol. 2017;96:137-8.

2. Oon HH, Chong WS, Oh CC. Simultaneous occurrence of papulonecrotic tuberculid and erythema induratum in an Asian woman. Skinmed. 2016;14:457-59.

3. Paller A, Mancini AJ. Bacterial, mycobacterial, and protozoal infections of the skin. Hurwitz Clinical Pediatric Dermatology: Textbook of skin disorders of childhood and adolescence. $5^{\text {th }}$ ed. New York, USA: Elsevier; 2016: 334-359.
4. Gupta V. Papulonecrotic tuberculid with scrofuloderma: An uncommon association. J Clin Diagn Res. 2015;9:WD03-4.

5. Singh SK, Rai T. Papulonecrotic tuberculid in a patient with pulmonary tuberculosis. Indian Dermatol Online J. 2014:5;72-3.

6. Dar NR,Raza N,Zafar O,Awan S.Papulonecrotic tuberculids associated with uveitis. J Coll Physicians Surg Pak. 2008;18:236-8.

7. Muto J, Kuroda K, Tajima S.Papular tuberculides post -BCG vaccination: Case report and review of literature in Japan. Clin Exp Dermatol. 2006;31:611-2.

8. Chen SC,Tao HY, Tseng HH. Papulonecrotic tuberculid -a rare skin manifestation in a patients with pulmonary tuberculosis. J Formos Med Assoc. 2000;99:857-9.

Copyright by Th Bijayanti Devi, et al. This is an open-access article distributed under the terms of the Creative Commons Attribution License, which permits unrestricted use, distribution, and reproduction in any medium, provided the original author and source are credited.

Source of Support: Nil, Conflict of Interest: None declared. 